\title{
Troxerutin attenuates cognitive decline in the hippocampus of male diabetic rats by inhibiting NADPH oxidase and activating the Nrf2/ARE signaling pathway
}

\author{
MING GAO ${ }^{1}$, YAXING KANG ${ }^{1}$, LIHUI ZHANG ${ }^{1}$, HONGYAN LI $^{2}$, CHANGHUI QU $^{3}$, \\ XIAQING LUAN ${ }^{4}$, LIJUN LIU ${ }^{5}$ and SONGYUN ZHANG ${ }^{1}$
}

\author{
${ }^{1}$ Department of Endocrinology, The Second Hospital of Hebei Medical University, Shijiazhuang, Hebei 050000; \\ ${ }^{2}$ Department of Endocrinology, The First Shijiazhuang Hospital, Shijiazhuang, Hebei 050011; ${ }^{3}$ Department of Endocrinology, \\ The Affiliated Lianyungang Oriental Hospital of Xuzhou Medical University, Lianyungang, Jiangsu 222042; \\ ${ }^{4}$ Department of Gastroenterology, Pipeline Bureau Hospital of Langfang, Langfang, Hebei 065000; \\ ${ }^{5}$ Department of Hematology, The Affiliated Hospital of Chengde Medical College, Chengde, Hebei 067020, P.R. China
}

Received January 30, 2020; Accepted May 25, 2020

DOI: $10.3892 / \mathrm{ijmm} .2020 .4653$

\begin{abstract}
Diabetes-associated cognitive decline is a recently identified a potential complication of diabetes. The present study was designed to examine the effects of troxerutin, a potent antioxidant, on cognitive function in rats with streptozotocin-induced diabetes and to further explore the potential underlying mechanisms. Cognitive functions were investigated by the Morris water maze test. The malondialdehyde (MDA) level and superoxide dismutase (SOD) activity in the hippocampus were assessed as the parameters of oxidative stress. Subunits of the NADPH oxidase (NOX) expression and nuclear factor erythroid 2-related factor 2/antioxidant responsive element (Nrf2/ARE) signaling pathway were detected to explore the potential underlying mechanisms. The water maze test revealed that troxerutin significantly improved cognitive impairment in diabetic rats. Troxerutin treatment attenuated oxidative stress in the hippocampus of diabetic rats, as evidenced by the decreased MDA level and the increased SOD activity. Moreover, troxerutin activated the Nrf2/ARE signaling pathway via Nrf2 nuclear translocation in the cells in the hippocampus of diabetic
\end{abstract}

Correspondence to: Professor Songyun Zhang, Department of Endocrinology, The Second Hospital of Hebei Medical University, 215 Heping West Road, Xinhua, Shijiazhuang, Hebei 050000, P.R. China

E-mail: zsy_hmu@126.com

Abbreviations: MDA, malondialdehyde; SOD, superoxide dismutase; LA, $\alpha$-lipoic acid; STZ, streptozotocin; Nrf2, nuclear factor-E2-related factor-2; HO-1, heme oxygenase-1; NQO1, NAD(P) H:quinone oxidoreductase; ARE, antioxidant response element; Keap1, kelch-like ECH associating protein 1; NADPH, nicotinamide adenine dinucleotide phosphate

Key words: troxerutin, diabetes, cognitive decline, hippocampus, oxidative stress, $\mathrm{Nrf} 2$ rats. Troxerutin elevated the expression levels of the antioxidant enzymes, heme oxygenase-1 (HO-1) and NAD(P)H:quinone oxidoreductase (NQO1), and decreased the expression levels of the NOX subunits, gp91phox, p47phox and p22phox. On the whole, these findings demonstrate that troxerutin exerts neuroprotective effects against diabetes-associated cognitive decline by suppressing oxidative stress in the hippocampus of rats with streptozotocin-induced diabetes. Troxerutin may thus prove to be a potential therapeutic medicine for the treatment of diabetes-associated cognitive decline.

\section{Introduction}

Diabetes mellitus is a common chronic endocrine disorder characterized by hyperglycemia, resulting from insulin resistance or insufficiency. Currently, it is estimated that there are 382 million individuals with diabetes worldwide, which is predicted to increase by $55 \%$ by 2035 (1). Recently, cognitive impairment was identified as a potential complication of diabetes and a major pathway developing into dementia (2). Mijnhout et al proposed a specific term diabetes encephalopathy to clarify the characteristics of this disorder (3).

The exact pathophysiology of diabetic encephalopathy is not yet completely understood. However, it is likely that vascular disease, hypoglycemia, insulin resistance and hyperglycemia play significant roles in the development of diabetic encephalopathy (4). Previous studies by the authors have demonstrated that the hippocampus in a diabetes model manifests dysfunction in modulating inflammation, oxidative response and synaptic plasticity (5-7). The direct glucose toxicity caused by hyperglycemia in neurons is mainly due to increased intracellular glucose oxidation, which leads to the excessive production of free radicals $(8,9)$. Oxidative stress has been implicated in the development of diabetic complications, including diabetic retinopathy, nephropathy, peripheral neuropathy, and cardiovascular disease (10-13). Due to the high requirement of oxygen and the existence of abundant unsaturated lipids, the brain is vulnerable to free radical attacks, which leads to neuronal cell death, 
autophagy and inflammation (14,15). A substantial amount of evidence has revealed that enhanced oxidative stress in types 1 and 2 diabetes mediates diabetic neuropathy and cognitive deficits (16-19). Nicotinamide adenine dinucleotidephosphate (NADPH) oxidase is a major source of superoxide, which is a precursor of reactive oxygen species (ROS) (20). Increased NADPH oxidase (NOX) activity underlines the free radical overproduction in oxidative stress (21). The major mechanism of cells combating oxidative injury is the management of the nuclear factor erythroid 2-related factor 2/antioxidant responsive element (Nrf2/ARE) signaling pathway, which regulates the transcription and expression of multiple phase II antioxidant enzymes, including heme oxygenase-1 (HO-1) and NAD(P)H: Quinone oxidoreductase-1 (NQO1) (22).

Troxerutin, a trihydroxyethylated derivative of the natural bioflavonoid, rutin, is extracted from Sophora japonica. It has previously been demonstrated that troxerutin possesses a variety of beneficial activities, including antioxidant, anti-inflammatory and anti-thrombotic abilities (23). Moreover, troxerutin has been shown to exert a protective effect against D-gal-induced inflammation in diabetic cardiomyopathy (24). Studies on the protective effects of troxerutin against oxidative stress-induced diabetes-associated cognitive decline are rare. The present study aimed to i) explore the protective effects of troxerutin on cognitive decline in rats with streptozotocin (STZ)-induced diabetes; ii) investigate the link between troxerutin treatment and oxidative stress in the hippocampus; and iii) determine the role of NOX activity and the Nrf2/ARE signaling pathway in balancing cell homeostasis.

\section{Materials and methods}

Animals. A total of 50 male specific-pathogen free (SPF) Sprague-Dawley rats (weighing, 150-170 g) at the age of 10 weeks, were purchased from Beijing Vital River Laboratory Animal Technology. The justification of male rats was based on the relatively longer distance between the urethral orifice and genitals compared with female rats. The animals were housed in individual cages under a controlled environment (12:12 $\mathrm{h}$ day/night cycle; temperature, $22 \pm 1^{\circ} \mathrm{C}$ ) with free access to food and water. All experimental procedures followed the Guide for the Care and Use of Laboratory Animals. All protocols for animal treatment were approved by the Animal Ethics Committee of Hebei Medical University. The animals were allowed to acclimatize to the laboratory conditions for 2 weeks prior to the experiment.

Drugs. Troxerutin was purchased from Shanghai Jingchun Biotechnology Co., Ltd. $\alpha$-lipoic acid (LA) was purchased from Stada Arzneimittel AG.

Experimental design. The rat model of diabetes was established by a single intraperitoneal injection of STZ (Sigma-Aldrich; Merck) at doses of $60 \mathrm{mg} / \mathrm{kg}$ for 12-h fasted rats at the age of 12 weeks. STZ is a glucosamine-nitrosourea compound and alkylating agent that is particularly toxic to islet $\beta$-cells. STZ is widely used to induce type 1 diabetes in mice or rats by using a large dose on an empty stomach. A single intravenous injection of STZ into fasted rats at dose of $50-75 \mathrm{mg} / \mathrm{kg}$ body weight will cause the necrosis of $\beta$-cells followed by $\beta$-cell loss and the atrophy of islets (25). It will then lead to a decrease in insulin levels, hyperglycemia and an increase in serum free-fatty acids. STZ was dissolved in $0.1 \mathrm{mmol} / 1$ sodium citrate buffer ( $\mathrm{pH} 4.4)$. The rats in the normal control group received an intraperitoneal injection of sodium citrate buffer. The blood glucose levels of the caudal vein were measured after $72 \mathrm{~h}$ using a portable glucose meter (ACCU-CHEK Performa, Roche Diagnostics). The rat model of diabetes was successfully established once the blood glucose levels exceeded $16.7 \mathrm{mmol} / \mathrm{l}$ for 2 consecutive days. Body weight and fasting blood glucose (FBG) levels of the rats were recorded once each week. At 4 weeks after the STZ injection, 40 rats with diabetic cognitive dysfunction and 10 normal control rats were well prepared and evaluated.

The diabetic rats were randomly divided into 4 groups ( $n=10$ in each group) as follows: i) The diabetes control group (DC group), in which diabetic rats were left without any treatment; ii) diabetes and saline-treated group (DN group), in which diabetic rats were intraperitoneally injected with saline; iii) diabetes with LA treatment group (DL group), in which diabetic rats were injected intraperitoneally with LA at doses of $60 \mathrm{mg} / \mathrm{kg} / \mathrm{day}$; iv) diabetes with troxerutin treatment group (DT group), in which diabetic rats were injected intraperitoneally with troxerutin at doses of $150 \mathrm{mg} / \mathrm{kg} / \mathrm{day}$. Moreover, the normal control rats were injected intraperitoneally with physical saline at the same volume as the NC group $(n=10)$. The treatment was administered once a day for 6 weeks.

Morris water maze test. Spatial learning and memory in each group were analyzed using the Morris water maze for 5 consecutive days following the 6-week intervention. The Morris water maze test is a convenient and classical method with which to assess cognitive function in rodents. The apparatus was composed of a circular water pool $(150 \mathrm{~cm}$ in diameter and $60 \mathrm{~cm}$ high), containing various prominent visual cues, with opaque water kept at $24-26^{\circ} \mathrm{C}$. A transparent platform was hidden $1 \mathrm{~cm}$ below the water surface in one quadrant. For each trial, the latency to escape from water of each rat was calculated. A probe test, in which the hidden platform was removed, was conducted after the last trial on training day 5. The probe test was performed and the rats were allowed to swim freely for $60 \mathrm{sec}$ with the platform absent. The time spent in the target quadrant and the times of platform crossing were measured. All data were recorded using a visual tracking system (SuperMaze software, Shanghai Xinruan Information Technology, Co., Ltd.).

Biochemical analysis. Rats were sacrificed following anesthesia with $10 \%$ chloral hydrate $(300 \mathrm{mg} / \mathrm{kg}$, intraperitoneal injection). The rats were anaesthetized within $5 \mathrm{~min}$ after the chloral hydrate intraperitoneal injection. The anesthetized states of the rats were evaluated by the reaction of rats to external stimuli, such as clamping the root of the rat's tail. There were no signs of peritonitis, pain or discomfort following the intraperitoneal injection. The rats were then sacrificed by intracardiac puncture. The death of rats was confirmed by cardiac arrest. The brains of the rats were carefully and rapidly removed and washed with cold physiological saline. The hippocampus was immediately separated from the cerebrum on an iced plate and then stored at $-80^{\circ} \mathrm{C}$ for use in subsequent experiments. The hippocampal tissue was made into a tissue homogenate in ice-cold 
Table I. Primers used for RT-qPCR.

\begin{tabular}{lll}
\hline Gene name & \multicolumn{1}{c}{ Forward (5') primer } & \multicolumn{1}{c}{ Reverse (3') primer } \\
\hline gp91phox & 5'-CCTAAGATAGCGGTTGATGG-3' & 5'-GACTTGAGAATGGATGCGAA-3' \\
p47phox & 5'-GTCAGATGAAAGCAAAGCGA-3' & 5'-CATAGTTGGGCTCAGGGTCT-3' \\
p22phox & 5'-TCTGGGAGCAACACCTTGGAAAC-3' & 5'-AGAAGCCAAGTGAGGCCGAAGAG-3' \\
Nrf2 & 5'-GCGGCAACTTTATTCTTCCCTCT-3' & 5'-AGTCCCATTCACAAAAGACAAACA-3' \\
HO1 & 5'-GAAACAAGCAGAACCCAGTCTATG-3' & 5'-CAGCAGCTCAGGATGAGTACCTC-3' \\
NQO1 & 5'-CTGTGACAGCAAAATGAACGAGG-3' & 5'-ATAGCAACAAGTGGGTGGAGGAT-3' \\
$\beta$-actin & 5'-GGCATGGACTGTGGTCATGA-3' & 5'-TTCACCACCATGGAGAAGGC-3'
\end{tabular}

Nrf2, the nuclear factor-E2-related factor-2; HO1, heme oxygenase-1; NQO1, NAD(P)H:quinone oxidoreductase.

physiological saline solution, and centrifuged $\left(1,000 \times \mathrm{g}, 4^{\circ} \mathrm{C}\right.$, $10 \mathrm{~min}$ ) to remove particulates. The obtained supernatant was assayed. Superoxide dismutase (SOD) activity, and the malondialdehyde (MDA) and glutathione (GSH) levels were measured using a SOD Activity Assay kit, MDA assay kit and GSH assay kit supplied by Nanjing Jiancheng Bio-engineering according to the manufacturer's instructions.

Reverse transcription-quantitative PCR. Total RNA was isolated from the hippocampal tissues using TRIzol reagent (Invitrogen; Thermo Fisher Scientific, Inc.) and reverse transcribed into cDNA using the Takara RNA PCR kit (AMV) (Takara Biotechnology Co., Ltd.). Specific primers for the target transcripts listed in Table I were synthesized (Invitrogen; Thermo Fisher Scientific, Inc.). mRNA expression was quantified using the SYBR-Green PCR kit (SYBR ${ }^{\circledR}$ Premix Ex Taq ${ }^{\mathrm{TM}}$ II; Takara Biotechnology Co., Ltd.). The real-time detection was performed using the ABI prism 7900 RT-PCR System (Applied Biosystems). The results were normalized to the $\beta$-actin expression levels and the quantities of gene expression were calculated by the $2^{-\Delta \Delta \mathrm{Cq}}$ method (26). All experiments were performed in triplicate. The primers of the target genes are listed in Table I.

Western blot analysis. Hippocampal samples (100 mg) were homogenized and lysed on ice with cell lysis buffer (Ding Changsheng Biotechnology). The homogenates were centrifuged at $2,000 \mathrm{x}$ g or $5 \mathrm{~min}$ at $4^{\circ} \mathrm{C}$. The supernatants were then collected. Nuclear extraction reagent (Pierce; Thermo Fisher Scientific, Inc.) was used for the separation of the cytosolic extract (cytosol) and nuclear extract (nucleus). The protein concentration was determined using the Bradford assay (Pierce; Thermo Fisher Scientific, Inc.). Equal amounts of protein $(20 \mu \mathrm{g})$ were separated by $12 \%$ SDS-polyacrylamide gel electrophoresis, and transferred onto PVDF membranes. The membranes were blocked with $5 \%$ non-fat milk for $2 \mathrm{~h}$ and incubated overnight at $4^{\circ} \mathrm{C}$ with primary antibodies (rabbit anti-HO-1, Epitomics, cat. no. 2322-1, 1:800; anti-gp91phox, Santa Cruz Biotechnology, Inc., cat. no. sc-130543, 1:500; anti-p47phox, EMD Millipore, cat. no. 07-502, 1:500; anti-p22phox, Santa Cruz Biotechnology, Inc., cat. no. sc-130550, 1:200; anti-NQO1, Epitomics, cat. no. 2558-1, 1:1,000; anti-Nrf2, Epitomics, cat. no. 2178-1, 1:500; anti- $\beta$-actin, Santa Cruz Biotechnology, Inc., cat. no. sc-69879, 1:2,000; anti-histone H3, Santa Cruz Biotechnology, Inc., cat.
Table II. Effects of troxerutin on body weights and blood glucose levels in diabetic rats.

\begin{tabular}{lcc}
\hline Group & Body weight $(\mathrm{g})$ & $\begin{array}{c}\text { Fasting blood } \\
\text { glucose levels }(\mathrm{mmol} / \mathrm{l})\end{array}$ \\
\hline NC & $383.56 \pm 12.14$ & $7.63 \pm 0.24$ \\
DC & $268.67 \pm 8.41^{\mathrm{a}}$ & $28.29 \pm 0.88^{\mathrm{a}}$ \\
DN & $244.10 \pm 4.38^{\mathrm{a}}$ & $29.25 \pm 1.01^{\mathrm{a}}$ \\
DL & $263.72 \pm 6.74^{\mathrm{a}}$ & $30.54 \pm 0.84^{\mathrm{a}}$ \\
DT & $252.21 \pm 10.14^{\mathrm{a}}$ & $27.77 \pm 1.05^{\mathrm{a}}$ \\
\hline
\end{tabular}

$\mathrm{NC}$, normal control; DC, diabetic control; DN, diabetic + normal saline-treated; DL, diabetic + LA-treated; DT, diabetic + troxerutin-treated. ${ }^{\mathrm{a}} \mathrm{P}<0.05$ vs. $\mathrm{NC}$ group $(\mathrm{n}=10$, mean $\pm \mathrm{SD})$.

no. sc-517576, 1:1,000). The membranes were then incubated with goat anti-rabbit secondary antibody conjugated with horseradish peroxidase (cat. no. 7074, 1:15,000; Cell Signaling Technology, Inc.) for $1 \mathrm{~h}$ at room temperature. Immunoreactive proteins were detected using enhanced chemiluminescence (Applygen Technologies Inc.). The quantification of the detected bands was performed using ImageJ software $3.0(\mathrm{NIH})$. The data were standardized using $\beta$-actin (internal control of cytosolic protein) or histone H3 (internal control of nuclear protein).

Statistical analysis. All data are expressed as the means \pm SD. The data of the Morris water maze were analyzed by repeated measures ANOVA and Tukey's test as the post hoc test. The other data were analyzed by one-way ANOVA followed by the Tukey's test. All statistical analyses were performed using SPSS 23 software (SPSS, Inc.). A P-value $<0.05$ was considered to indicate a statistically significant difference.

\section{Results}

Effects of troxerutin on body weight and blood glucose level. Movement, drinking, eating, urine volume and the fur color of the NC rats were normal. By contrast, the diabetic rats exhibited polyuria, polydipsia, weight loss and a dull fur color. As shown in Table II, hyperglycemia persisted in the diabetic rats after the STZ injection. The rats in the DC 

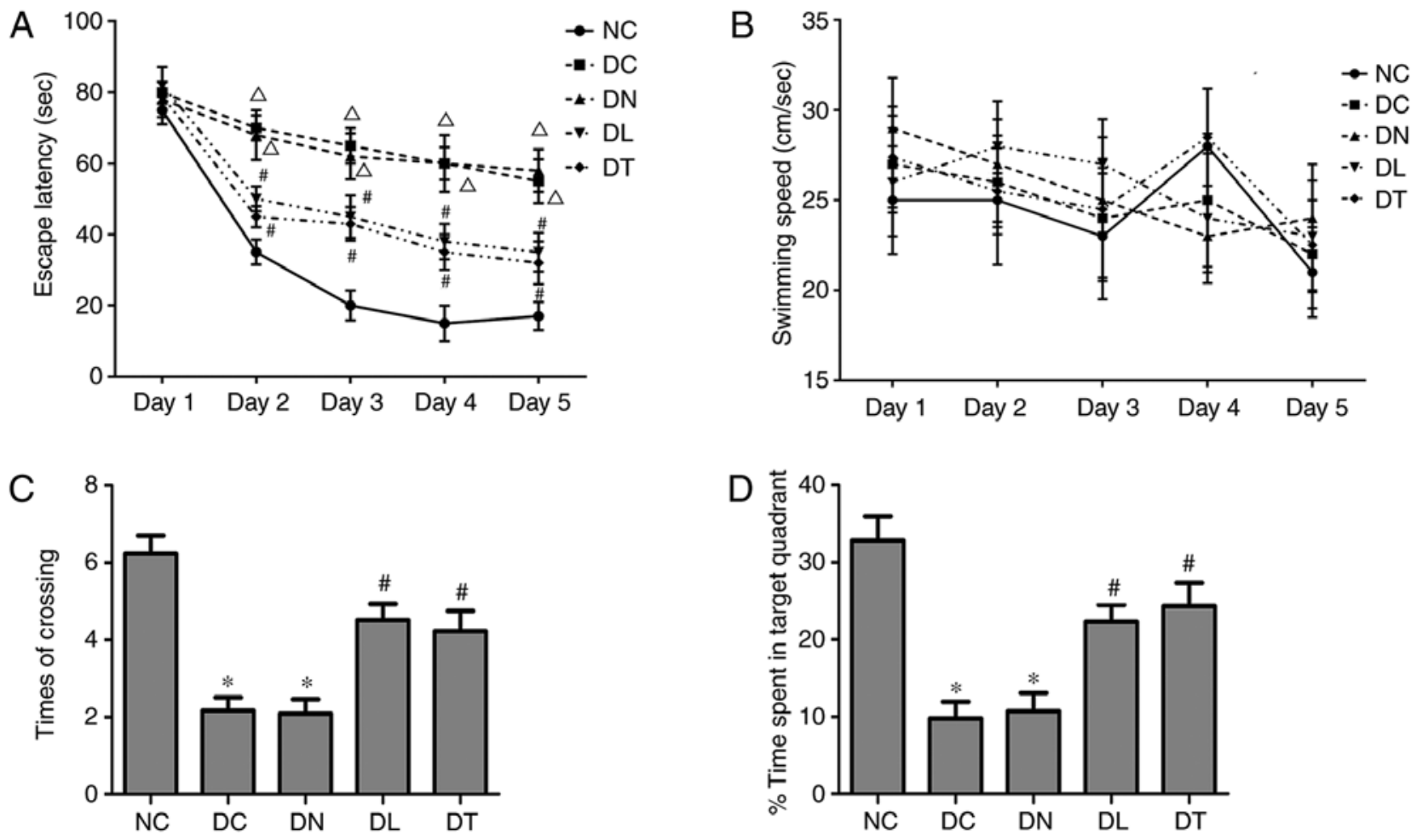

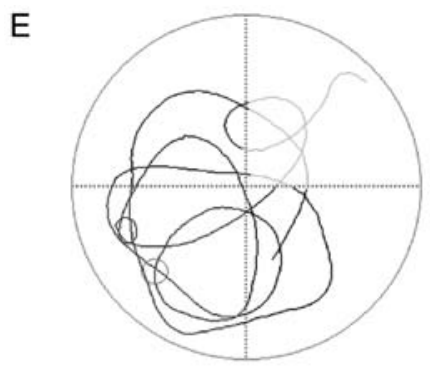

NC

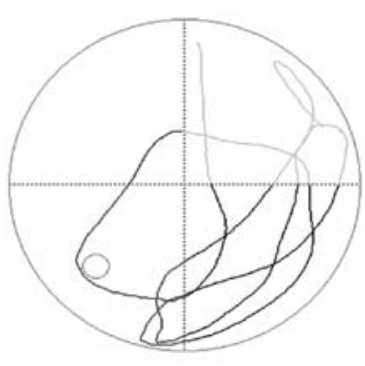

DC

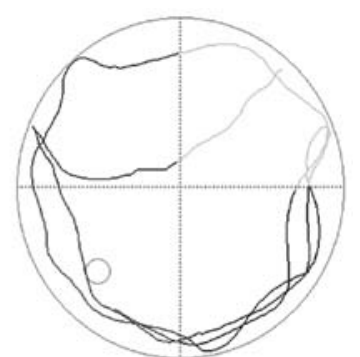

DN

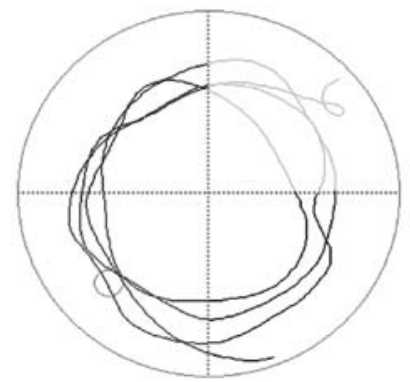

$\mathrm{DL}$

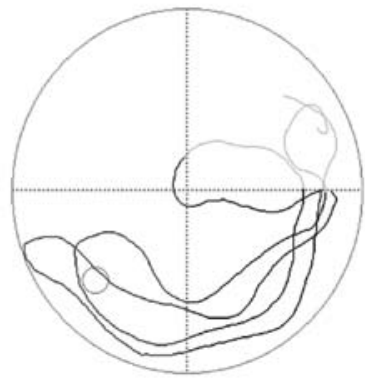

DT

Figure 1. Effects of troxerutin treatment on (A) escape latency and (B) swimming speed in the water maze test, and (C) times of crossing the platform area, as well as (D) the percentage of time spent in target quadrant during the probe trial of rats with STZ-induced diabetes. (E) Morris water maze path of each group. Data are presented as the means $\pm \mathrm{SD}, \mathrm{n}=10 .{ }^{\Delta} \mathrm{P}<0.01$ compared to NC group; ${ }^{*} \mathrm{P}<0.05$ compared to NC; ${ }^{*} \mathrm{P}<0.05$ compared to DC group. STZ, streptozotocin; NC group, normal blood glucose control rats; DC group, diabetic rats without any treatment; DN group, diabetes with saline treated group; DL group, diabetes with LA treated groups; DT group, diabetes with troxerutin treated group.

group was characterized by significantly increased FBG levels and a reduced body weight compared with those in the NC group $\left(\mathrm{F}_{(4,45)}=130.22, \mathrm{P}<0.05 ; \mathrm{F}_{(4,45)}=33.59, \mathrm{P}<0.05\right)$. The other diabetic groups exhibited no statistically significant differences in FBG and body weight compared with the DC group $(\mathrm{P}>0.05)$. Thus, troxerutin and LA treatment had no marked effect on the FBG level and body weight of the diabetic rats.
Effects of troxerutin on diabetes-induced cognitive deficits. Repeated measures ANOVA was used to compare the data between each group (Fig. 1A). The escape latency of DC and DN group increased significantly compared with the NC group $(\mathrm{P}<0.01)$. The escape latency of the DT and DL group decreased significantly compared with the DC group $(\mathrm{P}<0.05)$. No significant differences were observed in escape latency between the rats treated with troxerutin and LA ( $\mathrm{P}>0.05)$. 
A

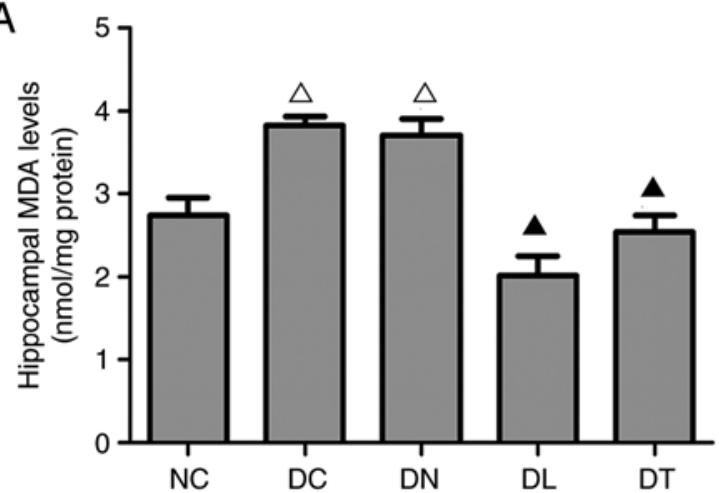

B

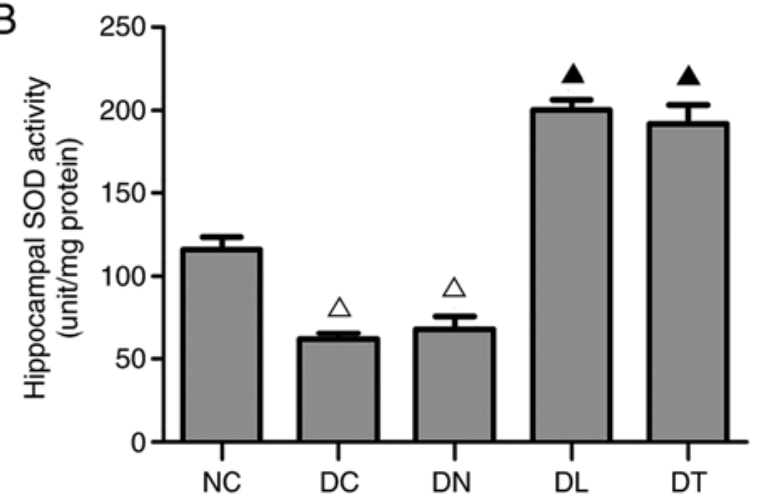

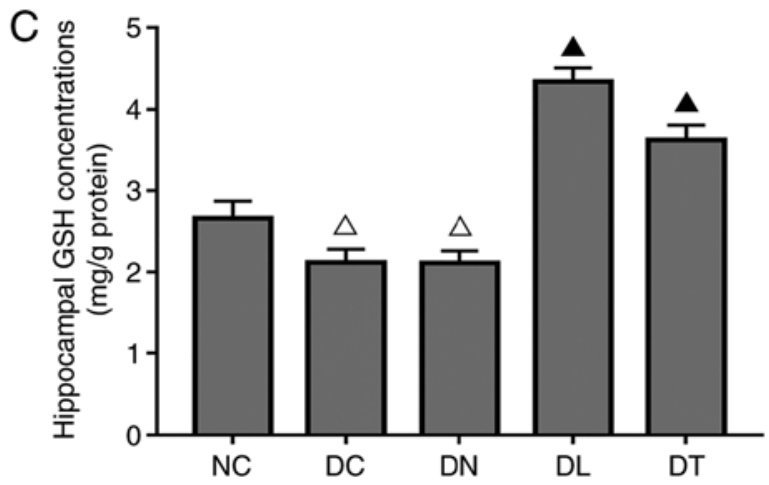

Figure 2. Effects of troxerutin treatment on (A) MDA levels, (B) SOD activities and (C) GSH concentration in the hippocampus of rats with STZ-induced diabetes. Data are presented as the means $\pm \mathrm{SD}, \mathrm{n}=3 .{ }^{\Delta} \mathrm{P}<0.01$ compared to $\mathrm{NC}$ group; ${ }^{\wedge} \mathrm{P}<0.01$ compared to DC group. $\mathrm{STZ}$, streptozotocin; $\mathrm{NC}$ group, normal blood glucose control rats; DC group, diabetic rats without any treatment; DN group, diabetes with saline treated group; DL group, diabetes with LA treated groups; DT group, diabetes with troxerutin treated group; MDA, malondialdehyde; SOD, superoxide dismutase; GSH, glutathione.

In a probe trial of the water maze test, the times of crossing the platform area (Fig. 1C) and percentage of time spent in the target quadrant (Fig. 1D) were significantly decreased in the DC rats compared with the NC rats. These performances were significantly improved by troxerutin or LA treatment $\left(\mathrm{F}_{(4,45)}=21.74, \mathrm{P}<0.05 ; \mathrm{F}_{(4,45)}=129.46, \mathrm{P}<0.05\right.$ respectively $)$, with no significant differences observed between the DT and DL groups $(\mathrm{P}>0.05)$. No significant differences were also observed in the swimming speed among the 5 groups ( $\mathrm{P}>0.05$, Fig. 1B), indicating that the motor deficits of rats did no contribute to the differences in escape latency, times of crossing and time spent in the target quadrant.

Effects of troxerutin on oxidative stress markers in the hippocampus of diabetic rats. As shown in Fig. 2, an increased SOD activity $\left(\mathrm{F}_{(4,25)}=427.22, \mathrm{P}<0.01\right)$ and $\mathrm{GSH}$ concentration $\left(\mathrm{F}_{(4,25)}=74.25, \mathrm{P}<0.01\right)$, alongside reduced MDA $\left(\mathrm{F}_{(4,25)}=189.12\right.$, $\mathrm{P}<0.01)$ levels were observed in the DL and DT groups, compared with the DC and DN groups.

Effects of troxerutin on the expression of NOX subunits in the hippocampus of diabetic rats. RT-qPCR and western blot analysis were performed to measure the expression of NOX subunits in the hippocampus, including gp91phox, p47phox and p22phox. As illustrated in Fig. 3E-G, STZ-induced diabetes increased the mRNA expression of gp91phox, p47phox and p22phox in the DC and DN groups, while troxerutin treatment attenuated the augmentation of gp91phox, p47phox and $\mathrm{p} 22$ phox expression in the diabetic rats $\left(\mathrm{F}_{(4,25)}=12.18, \mathrm{P}<0.01\right.$;
$\mathrm{F}_{(4,25)}=7.44, \mathrm{P}<0.05 ; \mathrm{F}_{(4,25)}=137.25, \mathrm{P}=0.01$, respectively). In addition, the cytosolic protein fractions of gp91phox, $\mathrm{p} 47 \mathrm{phox}$ and p22phox were significantly increased in the diabetic groups. Following troxerutin treatment, all these levels were decreased compared with the samples from the DC group $\left(\mathrm{F}_{(4,25)}=55.50, \mathrm{P}<0.01 ; \mathrm{F}_{(4,25)}=6.59, \mathrm{P}<0.05 ; \mathrm{F}_{(4,25)}=60.58\right.$, $\mathrm{P}<0.01$ ) (Fig. 3A-D).

Effects of troxerutin on the Nrf2/ARE signaling pathway in the hippocampus of diabetic rats. To determine the effects of troxerutin on the activation of the Nrf2/ARE signaling pathway in the model of diabetes-induced cognitive deficits, the hippocampal expression levels of $\mathrm{Nrf} 2$ and two well-known downstream phase II antioxidant enzymes, HO-1 and NQO1, were measured by western blot analsyis and RT-qPCR. As illustrated in Fig. 4H and I, STZ-induced diabetes significantly decreased the NQO1 and HO-1 mRNA expression levels. Troxerutin treatment resulted in a noticeably increased mRNA expression of NQO1 and HO-1 compared with the DC group $\left(\mathrm{F}_{(4,25)}=72.53, \mathrm{P}<0.01 ; \mathrm{F}_{(4,25)}=6.95, \mathrm{P}<0.05\right.$, respectively).

As regards the cytosolic protein fractions, the NQO1 and HO-1 levels were markedly decreased in the DC group compared with those in the NC group. Troxerutin resulted in noticeably increased cytosolic protein fractions of NQO1 and HO-1 compared with those in the DC group $\left(\mathrm{F}_{(4,25)}=86.86\right.$, $\mathrm{P}<0.01 ; \mathrm{F}_{(4,25)}=96, \mathrm{P}<0.01$ ) (Fig. 4A, E and F).

In addition, STZ-induced diabetes significantly inhibited the nuclear translocation of Nrf2. This inhibitory effect of 


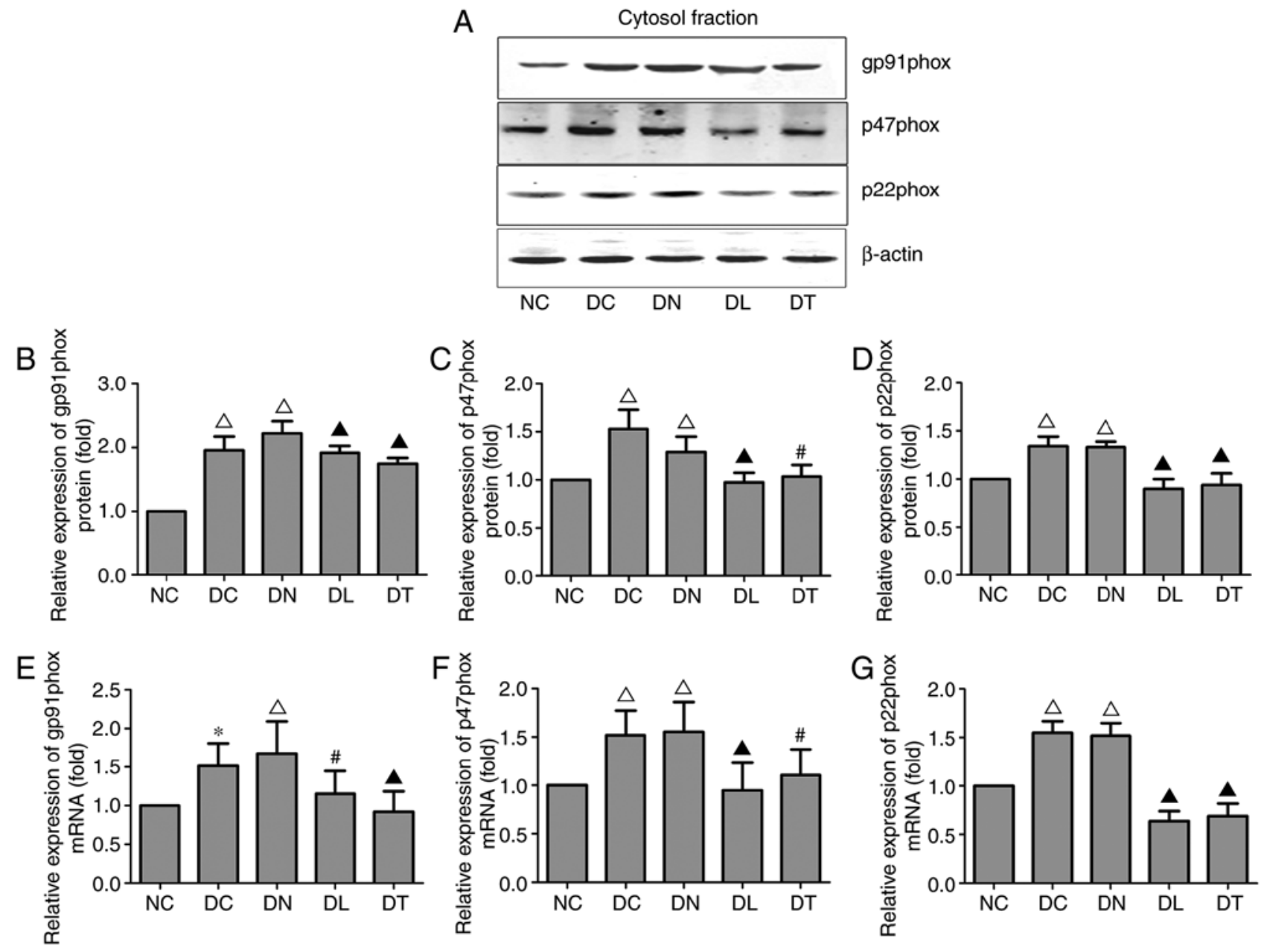

Figure 3. Effects of troxerutin treatment on the expression levels of NOX subunits in rats with STZ-induced diabetes. (A) Representative western blot for gp91phox, p47phox and p22ohox in the cytosolic protein fraction of hippocampal tissue. $\beta$-actin was used as a loading control. (B-D) The relative protein expression levels of NOX subunits are expressed as the ratio to $\beta$-actin. (E-G) Relative mRNA levels of NOX subunits. Experiments were performed on 3 independent hippocampal preparations $(\mathrm{n}=3)$. ${ }^{*} \mathrm{P}<0.05$ compared to $\mathrm{NC} ;{ }^{\Delta} \mathrm{P}<0.01$ compared to $\mathrm{NC} ;{ }^{\#} \mathrm{P}<0.05$ compared to $\mathrm{DC} ;{ }^{\wedge} \mathrm{P}<0.01$ compared to $\mathrm{DC}$ group. STZ, streptozotocin; NC group, normal blood glucose control rats; DC group, diabetic rats without any treatment; DN group, diabetes with saline treated group; DL group, diabetes with LA treated groups; DT group, diabetes with troxerutin treated group; MDA, malondialdehyde; SOD, superoxide dismutase; GSH, glutathione.

Nrf2 was reversed by troxerutin. Consistent with the changes observed in Nrf2 cytosolic and nuclear protein expression $\left(\mathrm{F}_{(4,25)}=52.35, \mathrm{P}<0.01 ; \mathrm{F}_{(4,25)}=159.36, \mathrm{P}<0.01\right)$ (Fig. 4A-D), the mRNA level of Nrf2 was markedly increased in the DT group compared with the DC group $\left(\mathrm{F}_{(4,25)}=69.62, \mathrm{P}<0.01\right)$ (Fig. $\left.4 \mathrm{G}\right)$. These results collectively demonstrate that troxerutin plays a protective role in the suppression of oxidative stress via the regulation of Nrf2/ARE signaling pathway. In Fig. 5, the schematic diagram demonstrates the mechanism of troxerutin in relieving the oxidative stress in hippocampus of diabetic rats.

\section{Discussion}

Diabetes mellitus is associated with an increased risk of dementia (2). The diabetes- associated cognitive decline is an increasing complication of diabetes (27). Accumulating studies have indicated that oxidative stress and inflammation response induced by chronic hyperglycemia can trigger the degenerative pathways leading to diabetes-associated cognitive decline $(11,19,28,29)$. In the present study, it was demonstrated that troxerutin exerted protective effects against cognitive impairment in rats with STZ-induced diabetes. The rats with STZ-induced diabetes exhibited typically increased blood glucose levels and a decreased body weight, as well as cognitive decline at the age of 22 weeks. Troxerutin treatment alleviated diabetes-induced oxidative damage, learning and memory loss in the hippocampus. At the molecular level, the administration of troxerutin reduced the activity of NOX, which is a major source of ROS. Moreover, it also demonstrated that troxerutin enhanced the Nrf2/ARE signaling pathway in hippocampus of STZ-induced diabetic rats, regulating the expression of $\mathrm{HO}-1$ and NQO1.

LA(1,2-dithiolane-3-pentanoic) is a natural dithiol compound synthesized from octanoic acid, which has been reported to have antioxidant activities. It is also a potent antioxidant inhibiting ROS-induced damaging effects. The present study selected LA as a positive control for its ability to scavenge ROS (30). The selection of troxerutin was based on its outstanding pharmacological potentials in cardiovascular disease and cognitive deficits (24,31-33). Its role in protecting against diabetes-associated cognitive decline, however, remains unclear. It is well known that the Morris water maze is a gold 
A

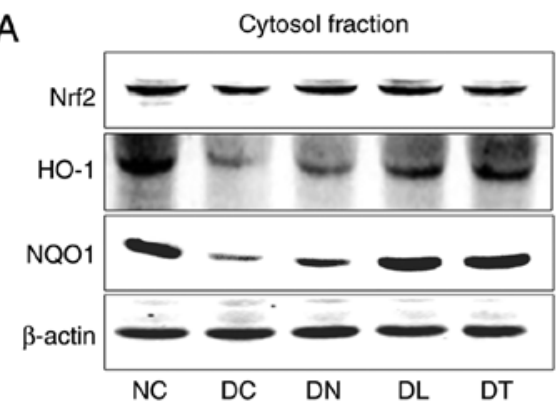

B
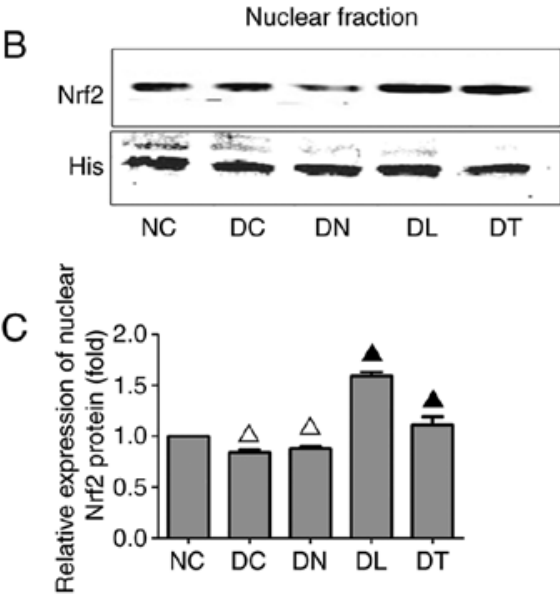
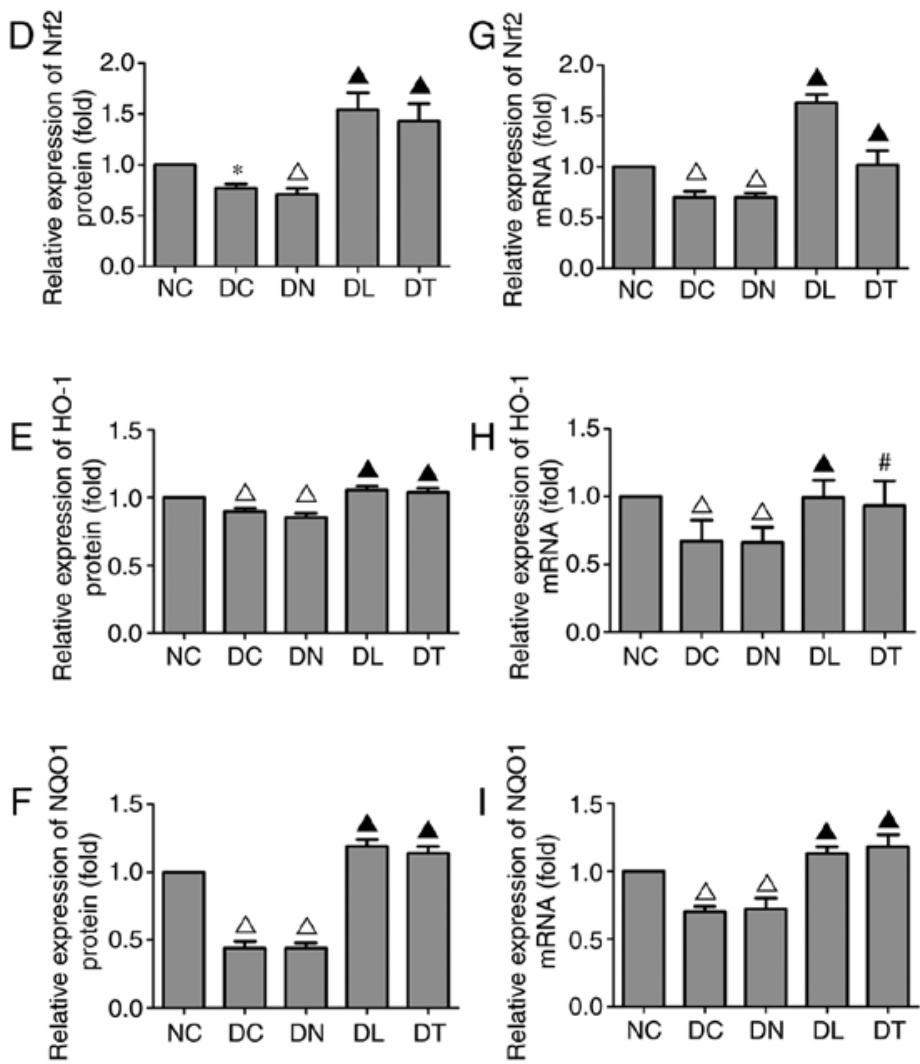

Figure 4. Effects of troxerutin treatment on the activation of the Nrf2/ARE signaling pathway. (A) Representative western blot of Nrf2, HO-1 and NQO1 in the cytosolic protein fraction of hippocampal tissue. (B) Representative western blot for Nrf2 in the nuclear fraction of hippocampal tissue. The relative protein expression of (C) nuclear Nrf2 and (D) cytosolic Nrf2, as well as the (G) mRNA level of Nrf2 were analyzed. Relative mRNA and cytosolic protein levels of $(\mathrm{E}$ and $\mathrm{H}$ ) HO-1 and ( $\mathrm{F}$ and $\mathrm{I}) \mathrm{NQO}$ are expressed as the ratio to $\beta$-actin. Experiments were performed on 3 independent hippocampal preparations ( $\mathrm{n}=3$ ). ${ }^{*} \mathrm{P}<0.05$ compared to $\mathrm{NC} ;{ }^{\Delta} \mathrm{P}<0.01$ compared to $\mathrm{NC} ;{ }^{*} \mathrm{P}<0.05$ compared to $\mathrm{DC} ;{ }^{\Delta} \mathrm{P}<0.01$ compared to DC group. NC group, normal blood glucose control rats; DC group, diabetic rats without any treatment; DN group, diabetes with saline treated group; DL group, diabetes with LA treated groups; DT group, diabetes with troxerutin treated group; MDA, malondialdehyde; SOD, superoxide dismutase; GSH, glutathione.

criterion for spatial learning and memory in rodents (34). In the present study, an increased escape latency, decreased times of crossing the platform area and the percentage of time spent in the target quadrant suggested that diabetes significantly impaired learning and memory performance, which is consistent with the findings of previous studies $(23,35)$. It was found that troxerutin treatment significantly improved the cognitive function of diabetic rats.

The elevation of the MDA level was assessed as an index for lipid peroxidation-induced oxidative damage. In addition, the activity of the antioxidant enzyme, SOD, was measured to evaluate the antioxidant response in hippocampus of diabetic rats. GSH is a major component of redox regulation. It has been shown in a previous study that the GSH concentration in the hippocampus of STZ-treated rats is reduced at hyperglycemia, but is normalized upon acute glycemic restoration to control levels (36).

In the present study, the increase in the MDA levels together with a decrease in SOD and GSH levels suggest that chronic hyperglycemia induced cognitive impairment through oxidative stress occurring in the hippocampus. Troxerutin decreased the MDA level, and increased SOD activity and the GSH level in the hippocampus of diabetic rats. Consequently, it ameliorated the diabetes-associated cognitive decline by reducing oxidative stress and enhancing antioxidant defenses in the hippocampus. Notably, LA exerted more potent suppressive effects on the MDA level, which is consistent with the findings of previous studies on the effects of LA on lipid peroxidation $(30,37)$.

NOX is a prooxidant enzyme that catalyzes the oxidation of NADPH, playing an important role in generating superoxide, particularly in neuronal pathologies. Indeed, previous studies have demonstrated that superoxide production by NOX underlies cognitive impairment following cerebral ischemia and traumatic brain injury $(38,39)$. NOX consists of membrane subunit proteins (p22phox and gp91phox) and cytosolic subunit proteins (p47phox, p67phox, p40phox). The cytosolic subunit proteins were significantly elevated with the progression of Alzheimer's disease (40). The increased expression of NOX membrane subunit p22phox underlies long-term memory loss induced by ROS overproduction in anesthetic exposure (41). Moreover, the knockdown of NOX subunit p47phox has been shown to increase spatial learning and memory in mice (42). The findings of the present study are in agreement with those of previous studies, demonstrating that troxerutin exerts antioxidant effects by reducing gp91phox, p47phox and p22phox expression levels in the hippocampus of diabetic rats. These data indicated that the upregulation of NOX in the hippocampus and the increase in NOX-associated redox pathways may participate in the early pathogenesis and contribute to the progression of diabetes-associated cognitive decline. 


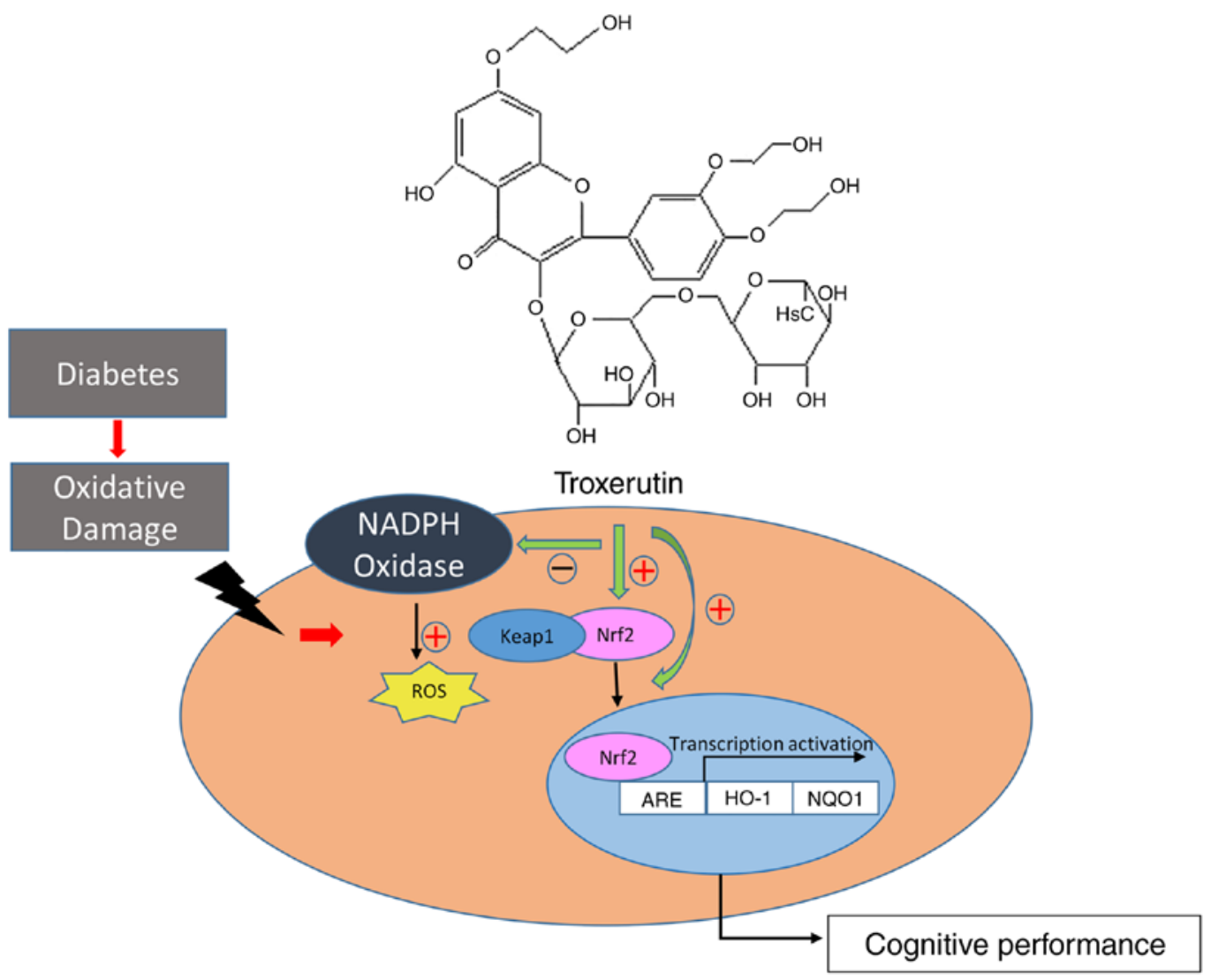

Figure 5. Schematic diagram illustrating the protective effects of troxerutin against diabetes-induced cognitive impairment and oxidative stress in the rat hippocampus. NADPH, nicotinamide adenine dinucleotide phosphate; ROS, reactive oxygen species; Nrf2, the nuclear factor-E2-related factor-2; HO1, heme oxygenase-1; NQO1, NAD(P)H:quinone oxidoreductase; ARE, antioxidant response element; Keap1, kelch-like ECH associating protein 1.

The Nrf2/ARE signaling pathway plays a key role in regulating transcription of the genes encoding endogenous antioxidant enzymes, such as NO-1, NQO1 and SOD (43). The present study demonstrated that the intracellular Nrf2 mRNA and protein levels in hippocampus increased with troxerutin followed STZ-induced hyperglycemia. When cells are exposed to free radicals, Nrf2 disassociates from its cytosolic inhibitor, Keap-1 and translocates to the nucleus, where it combines with the ARE (the promoter region of many phase II enzymes) (44-46). The results indicated that increased nuclear Nrf2 protein along with elevated HO-1 and NQO1 expression may be the underlying mechanism of troxerutin mitigating diabetes-associated cognitive decline.

Additionally, it was found LA treatment exerted more porent effects on promoting Nrf2 transcription and nuclear translocation. Although the diabetic rats benefited more from LA treatment, no statistically significant differences were observed in spatial learning and memory performances between the LA- and troxerutin-treated diabetic rats. This may due to other biological activities of troxerutin, such as anti-inflammatory, anti-thrombotic and anti-fibrinolytic effects.

In conclusion, the present study demonstrates that troxerutin prevents diabetes-associated cognitive decline in rats with STZ-induced diabetes by suppressing oxidative stress through the inhibition of NOX and the Nrf2/ARE signaling pathway. However, the present study only performed experiments on an animal model. The precise conclusions drawn warrant further verification in in vitro cell experiments. These findings however suggest that troxerutin may have therapeutic potential for use in protecting against cognitive dysfunction resulting from diabetes. Further studies are required to explore the interaction between NOX and the Nrf2/ARE signaling pathway.

\section{Acknowledgements}

Not applicable.

\section{Funding}

The present study was supported by the Natural Science Foundation of Hebei, China (grant no. H2018206320) and Health and Traditional Medicine Administration Scientific Research Plan of Hebei, China (grant no. 2019119).

\section{Availability of data and materials}

All data generated or analyzed during this study are included in this published article or are available from the corresponding author on reasonable request.

\section{Authors' contributions}

The corresponding author SZ contributed to the design and conception of the study. MG contributed to the design of 
the study, performed the experiments, analyzed the data and prepared the manuscript for writing. YK established the animal model and performed the behavioral test. LZ contributed the experimental design and manuscript reviewing. HL and CQ performed the molecular biological analysis. XL and LL were involved in data analysis and figure editing. All authors have read and approved the final manuscript.

\section{Ethics approval and consent to participate}

All experimental procedures followed the Guide for the Care and Use of Laboratory Animals. All protocols for animal treatment were approved by the Animal Ethics Committee of Hebei Medical University.

\section{Patient consent for publication}

Not applicable.

\section{Competing interests}

The authors declare that they have no competing interests.

\section{References}

1. Guariguata L, Whiting DR, Hambleton I, Beagley J, Linnenkamp U and Shaw JE: Global estimates of diabetes prevalence for 2013 and projections for 2035. Diabetes Res Clin Pract 103: 137-149, 2014

2. Koekkoek PS, Kappelle LJ, van den Berg E, Rutten GE and Biessels GJ: Cognitive function in patients with diabetes mellitus: Guidance for daily care. Lancet Neurol 14: 329-340, 2015.

3. Mijnhout GS, Scheltens P, Diamant M, Biessels GJ, Wessels AM, Simsek S, Snoek FJ and Heine RJ: Diabetic encephalopathy: A concept in need of a definition. Diabetologia 49: 1447-1448, 2006.

4. Biessels GJ and Despa F: Cognitive decline and dementia in diabetes mellitus: Mechanisms and clinical implications. Nat Rev Endocrinol 14: 591-604, 2018.

5. Gao M, Ji S, Li J and Zhang S: DL-3-n-butylphthalide (NBP) ameliorates cognitive deficits and CaMKII-mediated long-term potentiation impairment in the hippocampus of diabetic $\mathrm{db} / \mathrm{db}$ mice. Neurol Res 41: 1024-1033, 2019.

6. Li J, Zhang S, Zhang L, Wang R and Wang M: Effects of L-3-n-butylphthalide on cognitive dysfunction and NR2B expression in hippocampus of streptozotocin (STZ)-induced diabetic rats. Cell Biochem Biophys 71: 315-322, 2015.

7. Zhang SY, Ji SX, Bai XM, Yuan F, Zhang LH and Li J: L-3-n-butylphthalide attenuates cognitive deficits in $\mathrm{db} / \mathrm{db}$ diabetic mice. Metab Brain Dis 34: 309-318, 2019.

8. Muriach M, Flores-Bellver M, Romero FJ and Barcia JM Diabetes and the brain: Oxidative stress, inflammation, and autophagy. Oxid Med Cell Longev 2014: 102158, 2014.

9. Cobley JN, Fiorello ML and Bailey DM: 13 reasons why the brain is susceptible to oxidative stress. Redox Biol 15: 490-503, 2008.

10. Yang H, Jin X, Kei Lam CW and Yan SK: Oxidative stress and diabetes mellitus. Clin Chem Lab Med 49: 1773-1782, 2011.

11. Elsherbiny NM, Zaitone SA, Mohammad HMF and El-Sherbiny M: Renoprotective effect of nifuroxazide in diabetes-induced nephropathy: Impact on NFкB, oxidative stress, and apoptosis. Toxicol Mech Methods 28: 467-473, 2018.

12. Aboualizadeh E, Ranji M, Sorenson CM, Sepehr R, Sheibani N and Hirschmugl CJ: Retinal oxidative stress at the onset of diabetes determined by synchrotron FTIR widefield imaging: Towards diabetes pathogenesis. Analyst 142: 1061-1072, 2017.

13. Ravassa S, Beaumont J, Huerta A, Barba J, Coma-Canella I, González A, López B and Díez J: Association of low GLP-1 with oxidative stress is related to cardiac disease and outcome in patients with type 2 diabetes mellitus: A pilot study. Free Radic Biol Med 81: 1-12, 2015.
14. Mecocci P, Boccardi V, Cecchetti R, Bastiani P, Scamosci M, Ruggiero $C$ and Baroni M: A long journey into aging, brain aging, and Alzheimer's disease following the oxidative stress tracks. J Alzheimers Dis 62: 1319-1335, 2018.

15. Maciejczyk M, Żebrowska $E$ and Chabowski A: Insulin resistance and oxidative stress in the brain: What's new? Int J Mol Sci 20: 874, 2019.

16. Kim J, Cho CH, Hahn HG, Choi SY and Cho SW: Neuroprotective effects of $\mathrm{N}$-adamantyl-4-methylthiazol-2-amine against amyloid $\beta$-induced oxidative stress in mouse hippocampus. Brain Res Bull 128: 22-28, 2017.

17. Tian X, Liu Y, Ren G, Yin L, Liang X, Geng T, Dang H and An R: Resveratrol limits diabetes-associated cognitive decline in rats by preventing oxidative stress and inflammation and modulating hippocampal structural synaptic plasticity. Brain Res 1650: 1-9, 2016.

18. Samarghandian S, Azimi-Nezhad M and Samini F: Ameliorative effect of saffron aqueous extract on hyperglycemia, hyperlipidemia, and oxidative stress on diabetic encephalopathy in streptozotocin induced experimental diabetes mellitus. Biomed Res Int 2014: 920857, 2014.

19. Gomaa AA, Makboul RM, Al-Mokhtar MA and Nicola MA: Polyphenol-rich Boswellia serrata gum prevents cognitive impairment and insulin resistance of diabetic rats through inhibition of GSK $3 \beta$ activity, oxidative stress and pro-inflammatory cytokines. Biomed Pharmacother 109: 281-292, 2019.

20. Bedard K and Krause KH: The NOX family of ROS-generating NADPH oxidases: Physiology and pathophysiology. Physiol Rev 87: 245-313, 2007.

21. Hu XF, Wang L, Xiang G, Lei W and Feng YF: Angiogenesis impairment by the NADPH oxidase-triggered oxidative stress at the bone-implant interface: Critical mechanisms and therapeutic targets for implant failure under hyperglycemic conditions in diabetes. Acta Biomater 73: 470-487, 2018.

22. Nguyen T, Nioi P and Pickett CB: The Nrf2-antioxidant response element signaling pathway and its activation by oxidative stress. J Biol Chem 284: 13291-13295, 2009.

23. Zhang S, Li H, Zhang L, Li J, Wang R and Wang M: Effects of troxerutin on cognitive deficits and glutamate cysteine ligase subunits in the hippocampus of streptozotocin-induced type 1 diabetes mellitus rats. Brain Res 1657: 355-360, 2017.

24. Najafi M, Noroozi E, Javadi A and Badalzadeh R: Anti-arrhythmogenic and anti-inflammatory effects of troxerutin in ischemia/reperfusion injury of diabetic myocardium. Biomed Pharmacother 102: 385-391, 2018.

25. Goyal SN, Reddy NM, Patil KR, Nakhate KT, Ojha S, Patil CR and Agrawal YO: Challenges and issues with streptozotocin-induced diabetes-A clinically relevant animal model to understand the diabetes pathogenesis and evaluate therapeutics. Chem Biol Interact 244: 49-63, 2016.

26. Livak KJ and Schmittgen TD: Analysis of relative gene expression data using real-time quantitative PCR and the 2(-Delta Delta C(T)) method. Methods 25: 402-408, 2001.

27. Rawlings AM, Sharrett AR, Schneider AL, Coresh J, Albert M, Couper D, Griswold M, Gottesman RF, Wagenknecht LE, Windham BG and Selvin E: Diabetes in midlife and cognitive change over 20 years: A cohort study. Ann Intern Med 161: 785-793, 2014.

28. Chung CC, Pimentel D, Jor'Dan AJ, Hao Y, Milberg W and Novak V: Inflammation-associated declines in cerebral vasoreactivity and cognition in type 2 diabetes. Neurology 85: 450-458, 2015.

29. Ward R and Ergul A: Relationship of endothelin-1 and NLRP3 inflammasome activation in HT22 hippocampal cells in diabetes. Life Sci 159: 97-103, 2016.

30. Shi XC, Jin A, Sun J, Yang Z, Tian JJ, Ji H, Yu HB, Li Y, Zhou JS, Du ZY and Chen LQ: $\alpha$-lipoic acid ameliorates n-3 highly-unsaturated fatty acids induced lipid peroxidation via regulating antioxidant defenses in grass carp (Ctenopharyngodon idellus). Fish Shellfish Immunol 67: 359-367, 2017.

31. Lu J, Wu DM, Zheng YL, Hu B, Cheng W, Zhang ZF and Li MQ: Troxerutin counteracts domoic acid-induced memory deficits in mice by inhibiting CCAAT/enhancer binding protein $\beta$-mediated inflammatory response and oxidative stress. J Immunol 190: 3466-3479, 2013.

32. Lu J, Wu DM, Zheng ZH, Zheng YL, Hu B and Zhang ZF: Troxerutin protects against high cholesterol-induced cognitive deficits in mice. Brain 134: 783-797, 2011.

33. $\mathrm{Yu} \mathrm{Y}$ and Zheng G: Troxerutin protects against diabetic cardiomyopathy through NF- $\mathrm{kB} / \mathrm{AKT} / \mathrm{IRS} 1$ in a rat model of type 2 diabetes. Mol Med Rep 15: 3473-3478, 2017. 
34. Morris R: Developments of a water-maze procedure for studying spatial learning in the rat. J Neurosci Methods 11: 47-60, 1984.

35. Zhang S, Yuan L, Zhang L, Li C and Li J: Prophylactic use of troxerutin can delay the development of diabetic cognitive dysfunction and improve the expression of Nrf2 in the hippocampus on STZ diabetic rats. Behav Neurol 2018: 8678539, 2018

36. Duarte JM, Carvalho RA, Cunha RA and Gruetter R: Caffeine consumption attenuates neurochemical modifications in the hippocampus of streptozotocin-induced diabetic rats J Neurochem 111: 368-379, 2009.

37. Uchendu C, Ambali SF, Ayo JO and Esievo KAN: Chronic co-exposure to chlorpyrifos and deltamethrin pesticides induces alterations in serum lipids and oxidative stress in Wistar rats: Mitigating role of alpha-lipoic acid. Environ Sci Pollut Res Int 25: 19605-19611, 2018.

38. Shi GX, Wang XR, Yan CQ, He T, Yang JW, Zeng XH, Xu Q, Zhu W, Du SQ and Liu CZ: Acupuncture elicits neuroprotective effect by inhibiting NAPDH oxidase-mediated reactive oxygen species production in cerebral ischaemia. Sci Rep 5: 17981, 2015.

39. Ferreira AP, Rodrigues FS, Della-Pace ID, Mota BC, Oliveira SM, Velho Gewehr Cde C, Bobinski F, de Oliveira CV, Brum JS Oliveira MS, et al: The effect of NADPH-oxidase inhibitor apocynin on cognitive impairment induced by moderate lateral fluid percussion injury: Role of inflammatory and oxidative brain damage. Neurochem Int 63: 583-593, 2013.

40. Ansari MA and Scheff SW: NADPH-oxidase activation and cognition in Alzheimer disease progression. Free Radic Biol Med 51: 171-178, 2011.
41. Sun Z, Satomoto M, Adachi YU, Kinoshita H and Makita K: Inhibiting NADPH oxidase protects against long-term memory impairment induced by neonatal sevoflurane exposure in mice. Br J Anaesth 117: 80-86, 2016.

42. Walton JC, Selvakumar B, Weil ZM, Snyder SH and Nelson RJ: Neuronal nitric oxide synthase and NADPH oxidase interact to affect cognitive, affective, and social behaviors in mice. Behav Brain Res 256: 320-327, 2013.

43. Ahmed SM, Luo L, Namani A, Wang XJ and Tang X: Nrf2 signaling pathway: Pivotal roles in inflammation. Biochim Biophys Acta Mol Basis Dis 1863: 585-597, 2017.

44. Shalaby YM, Menze ET, Azab SS and Awad AS: Involvement of Nrf2/HO-1 antioxidant signaling and NF- $\mathrm{NB}$ inflammatory response in the potential protective effects of vincamine against methotrexate-induced nephrotoxicity in rats: Cross talk between nephrotoxicity and neurotoxicity. Arch Toxicol 93: 1417-1431, 2019.

45. Zhang L, Guo Y, Wang H, Zhao L, Ma Z, Li T, Liu J, Sun M, Jian Y, Yao L, et al: Edaravone reduces $A \beta$-induced oxidative damage in SH-SY5Y cells by activating the Nrf2/ARE signaling pathway. Life Sci 221: 259-266, 2019.

46. Ren B, Yuan T, Diao Z, Zhang C, Liu Z and Liu X: Protective effects of sesamol on systemic oxidative stress-induced cognitive impairments via regulation of Nrf2/Keap1 pathway. Food Funct 9: 5912-5924, 2018. 